\title{
A Multiple-Microactuator System Using a High-Frequency Pressure Source
}

\author{
Kazuhiro Yoshida*, Satoshi Yamamotoํㅗ Sang In Eom and Shinichi Yokota \\ Precision and Intelligence Laboratory, Tokyo Institute of Technology, \\ R2-42, 4259 Nagatsuta-cho, Midori-ku, Yokohama 226-8503, Japan \\ 'Graduate School, Tokyo Institute of Technology, \\ R2-42, 4259 Nagatsuta-cho, Midori-ku, Yokohama 226-8503, Japan
}

(Received July 31, 2014; accepted March 24, 2015)

Key words: microactuator, piezoelectric valve, alternating pressure, high frequency, multipleactuator system

For a micromachine having multiple high-power microactuators such as an inpipe working micromachine, a multiple-microactuator system using a high-frequency pressure source was proposed and developed. The system consists of a high-frequency piezoelectric pressure source, hydraulic microactuator units, and pipes; the hydraulic microactuator unit consists of a bending rubber actuator with two chambers and two thin piezoelectric valves, each of which is connected to the corresponding chamber of the actuator. An alternating flow from the pressure source is rectified by the synchronous switching of the valves, and the bending rubber actuator bends step by step. Each hydraulic microactuator unit needs only one pipe for the supply and return of working fluid, and the small piping space is useful for multiple-actuator systems. In addition, owing to the high-response pressure source and valves, high position resolution and downsizing of the pressure source are realized. A thin piezoelectric valve and a highfrequency piezoelectric pressure source using piezoelectric bimorph disks were proposed, fabricated and experimentally characterized. Then, a 10-mm-long bending rubber actuator was fabricated and a large model system was constructed. Its operation was experimentally tested and characterized, yielding a maximum tip displacement of 3 $\mathrm{mm}$ and a maximum force of $12 \mathrm{mN}$.

\section{Introduction}

An in-pipe working micromachine ${ }^{(1-4)}$ for the maintenance of small-diameter $(\sim 10 \mathrm{~mm})$ pipelines requires multiple independent microactuators to perform forward travel, reverse travel, steering, manipulation of objects, and so forth, as shown in Fig. 1. Hydraulic microactuators can produce $10^{3}$ times higher power density than

${ }^{*}$ Corresponding author: e-mail: yoshida@pi.titech.ac.jp 
an electromagnetic actuator at $1 \mathrm{~mm}$ size $^{(5)}$ and have been developed. However, a conventional hydraulic microactuator needs two pipes for the supply and return of working fluid, and the piping space limits the number of installable microactuators. A conventional pneumatic actuator needs only one pipe to supply air utilizing the outside as a tank. However, the in-pipe working micromachine mentioned above cannot utilize the outside because it is often filled with the other gases or liquids of the pipelines.

To overcome this drawback, the utilization of an alternating flow has been investigated. Nishioka et al. proposed a multiple-pneumatic-actuator system using resonance valves. ${ }^{(6)}$ In the resonance valve, both the valve body and the valve seat are supported by springs and the valve can open at the resonance frequency. Each valve having its own resonance frequency can be controlled by adjusting the frequency of the alternating pressure. Kitagawa et al. developed a pneumatic pilot valve using sound pressure. ${ }^{(7)}$ In the pilot valve, a nozzle-flapper system whose flapper is excited by sound pressure at the resonance frequency was employed. However, both systems seem to have a limitation in the downsizing of the valves owing to the complex structures. Some of the authors have proposed a multiple-electro-rheological (ER) microactuator system using an alternating-pressure source, which rectifies an alternating flow from the pressure source by the synchronous switching of ER microvalves. ${ }^{(8)}$ The ER microvalve can be opened or closed by changing the apparent viscosity of an ER fluid (ERF) owing to its applied electric field. An actuator of the proposed system has only one pipe for the supply and return of the working fluid. In addition, the pipe diameter can be small because the alternating pressure can be transmitted by a low-viscosity fluid. A large model was fabricated and the validity was confirmed. However, the system has a problem of low position resolution owing to the large position fluctuation caused by a low driving frequency for the ER microvalves and the alternating-pressure source using a voice coil motor.

In this study, high-response valves and a high-response pressure source are developed using high-response piezoelectric elements, and the system performance using the components is experimentally characterized.

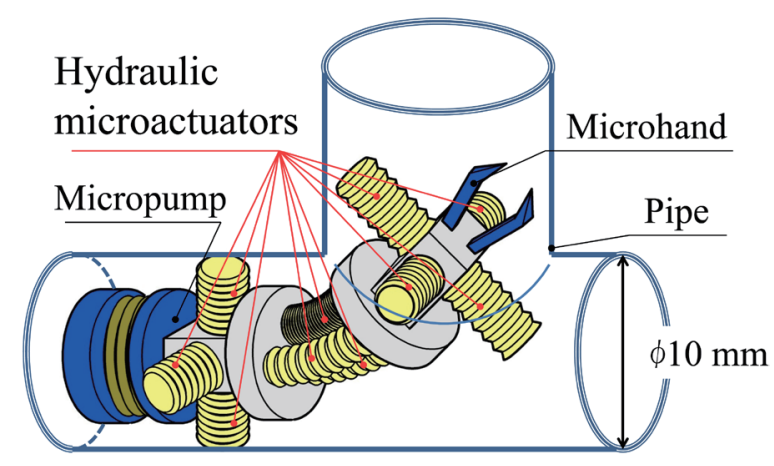

Fig. 1. (Color online) An example of a multiple-microactuator system. 


\section{Proposal of a Multiple Microactuator System Using a High-Frequency Pressure Source}

\subsection{Proposed multiple-microactuator system}

A conventional multiple-hydraulic-microactuator system is shown in Fig. 2(a). The system consists of a pressure source, hydraulic microactuator units, and pipes. The pressure source is composed of a pump and a relief valve to maintain the supply pressure. The hydraulic microactuator unit is composed of a hydraulic microactuator and two active valves. Varying the openings of the active valves makes it possible to control the internal pressure of the hydraulic microactuator, which finally controls the displacement of the actuator. However, each actuator needs two pipes for the supply and return of the working fluid, and thus it requires a large-volume piping system, especially when it is integrated into a multiple-actuator system, as shown in Fig. 2(a). To overcome this issue, we have proposed a new microactuator system that can significantly reduce the system volume.

The basic structure of the proposed system is shown in Fig. 2(b). The system consists of an alternating-pressure source, hydraulic microactuator units, and pipes. An alternating flow from the pressure source is rectified by the synchronous switching of the active valves, and the unidirectional flow is supplied to the hydraulic microactuators. Each hydraulic microactuator unit requires only one pipe for both the supply and return of the working fluid, which leads to the significant reduction of the piping space along with the proposed simple piping structure. It makes the proposed system suitable for a multiple-microactuator system. As the working fluid, a low-viscosity fluid such as water can be employed.

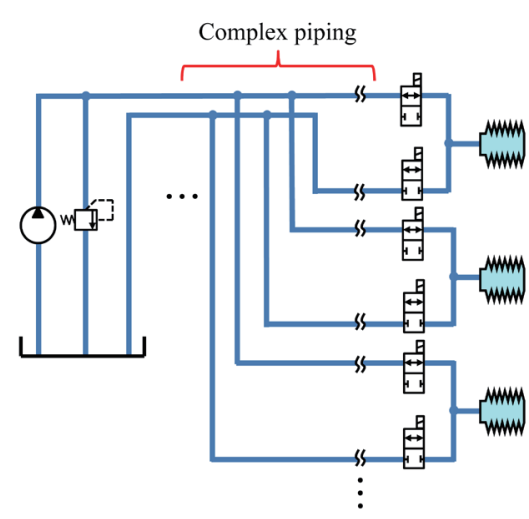

(a)

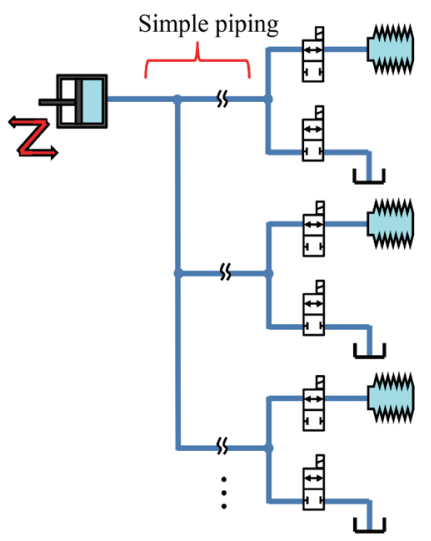

(b)

Fig. 2. (Color online) Multiple-microactuator systems. (a) Conventional system. (b) Proposed system. 
The basic structure of the actuator system shown in Fig. 2(b) is modified by replacing the actuator-tank system with a bending rubber actuator, which has two chambers enabling the bending motion of the actuator like a microfinger using a functional electroconjugate fluid (ECF), ${ }^{(9)}$ as shown in Fig. 3. In the multiple-actuator system, many hydraulic microactuator units are connected to an alternating-pressure source.

In the supply phase shown in Fig. 3(a), the active valve $A$ is opened and the active valve $B$ is closed so that the upper chamber is supplied with the working fluid. Accordingly, the upper chamber is expanded while the length of the lower chamber remains unchanged, which finally induces the downward motion of the bending rubber actuator by one step. In the successive return phase shown in Fig. 3(b), valve A is closed and valve B is opened so that the lower chamber is sucked and contracted while the length of the upper chamber no longer changes, which leads to the further stepwise downward motion of the actuator. By iterating these phases, the working fluid is moved from the lower chamber to the upper chamber, and the bending rubber actuator is bent downward step by step. When the open and close states are exchanged in valves A and B, the bending rubber actuator is bent upward. When both valves $\mathrm{A}$ and $\mathrm{B}$ are closed, the bending rubber actuator is stopped. By controlling the open and close states in valves $\mathrm{A}$ and $\mathrm{B}$, the motion of the bending rubber actuator can be controlled.

In the bending motion in one cycle shown in Fig. 3, the difference in volumes of the upper and lower chambers of the bending rubber actuator is changed by $2 \mathrm{~V}$, where $V$ is the displacement of the alternating-pressure source. As a primary approximation, assuming that the tip displacement change $\Delta x$ in one cycle is proportional to the volume difference $2 \mathrm{~V}$, the following equation is derived:

$$
U=\Delta x \cdot f=\alpha V f, \therefore\left\{\begin{array}{l}
\Delta x=U / f \\
V=U / \alpha f
\end{array}\right.
$$

where $f$ is the driving frequency, $U$ is the average tip velocity, and $\alpha$ is a constant. For

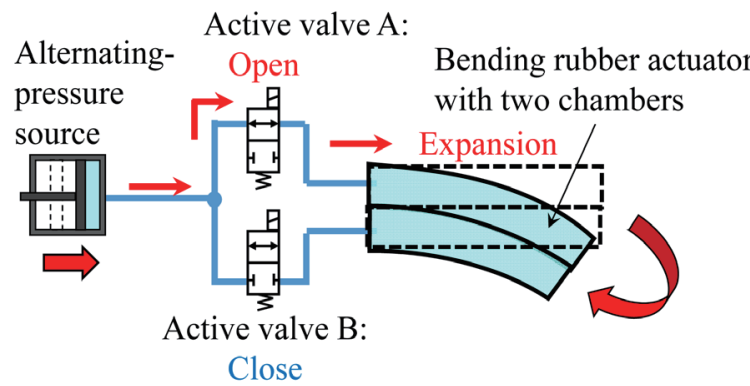

(a)

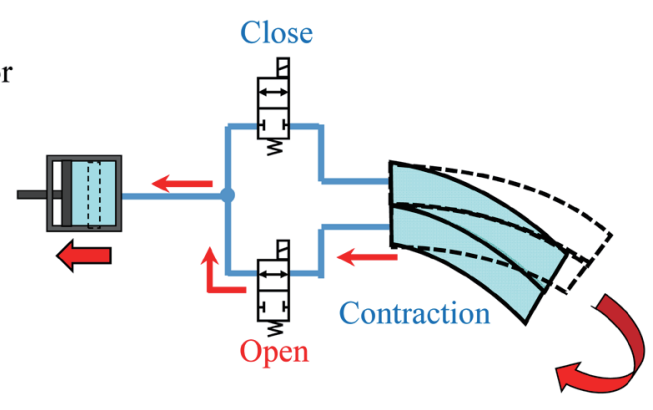

(b)

Fig. 3. (Color online) Proposed microactuator system using an alternating-pressure source. (a) Supply phase. (b) Return phase. 
the average tip velocity $U$, with increasing frequency $f$, the tip displacement change $\Delta x$ and the displacement $V$ are decreased, which results in the high resolution of the tip displacement and the downsizing of the alternating-pressure source.

In this study, a system using high-response active valves and an alternating-pressure source using high-response piezoelectric elements are proposed and developed. In the proposed system, the working fluid is water, and bias pressure is applied to prevent cavitation caused by negative gauge pressure.

\subsection{Theoretical analyses}

Theoretical analyses were performed to investigate the basic characteristics, especially for the inertia effect of the working fluid. For simplification, the basic singlehydraulic-microactuator system shown in Fig. 2(b) is modeled, as shown in Fig. 4(a). The system consists of an alternating-pressure source, an active valve, a hydraulic microactuator, and a pipe. The pressure source supplies pressure as

$$
p_{\mathrm{s}}(t)=P_{0} \sin \omega t
$$

with the bias pressure $P_{\mathrm{b}}$, where $P_{0}$ and $\omega=2 \pi f$ are the amplitude and angular frequency

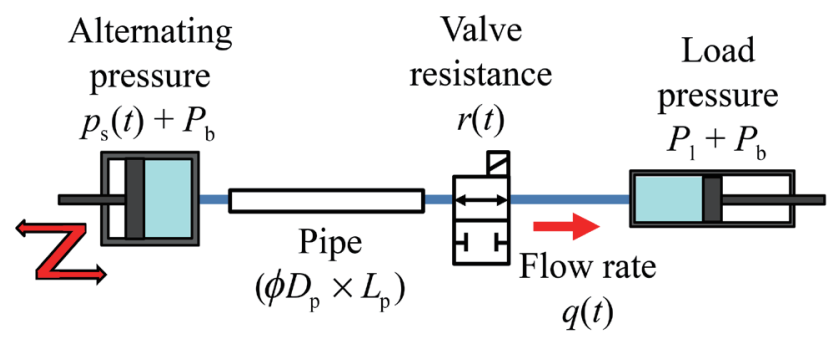

(a)

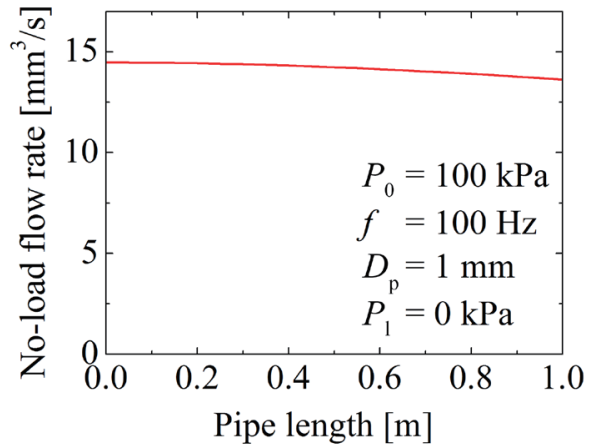

(b)

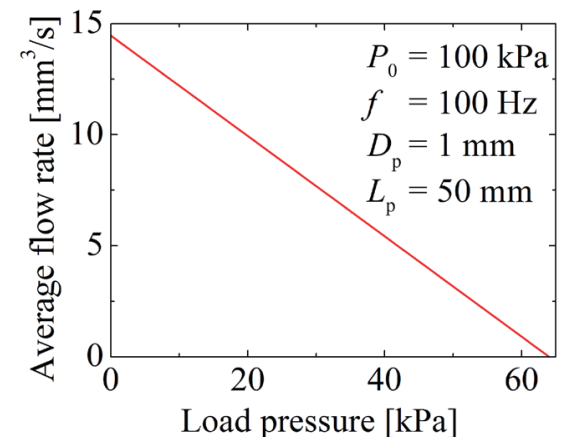

(c)

Fig. 4. (Color online) Theoretical analyses. (a) Model. (b) No-load flow rate as a function of pipe length. (c) Load characteristics. 
of the alternating pressure, respectively, and $t$ is time.

The valve resistance is expressed as

$$
r(t)= \begin{cases}R & \{n T \leq t<(n+0.5) T\} \\ \infty & \{(n+0.5) T \leq t<(n+1) T\}\end{cases}
$$

where $T=2 \pi / \omega$ and $n$ is an integer.

The pipe has the diameter $D_{\mathrm{p}}$, the length $L_{\mathrm{p}}$ and fluid inertance as

$$
L=\frac{4 \rho L_{\mathrm{p}}}{\pi D_{\mathrm{p}}^{2}}
$$

where $\rho$ is the density of the working fluid. The pipe resistance is neglected, because it is less than $2 \%$ of the valve resistance in these analyses. The load pressure $P_{1}$ is assumed to be constant with the bias pressure $P_{\mathrm{b}}$.

In the model shown in Fig. 4(a), the following equation is derived:

$$
L \frac{d q(t)}{d t}+r(t) q(t)+P_{1}=p_{\mathrm{s}}(t)
$$

where $q(t)$ is the flow rate.

By solving eq. (5), the average flow rate $q_{\mathrm{a}}$ is obtained as

$$
\begin{gathered}
q_{\mathrm{a}}=\frac{1}{T} \int_{n T}^{(n+1) T} q(t) d t=q_{\mathrm{a}, \max }\left(1-\frac{P_{1}}{P_{1, \max }}\right), \\
q_{\mathrm{a}, \max }=\frac{2+\omega^{2} T_{0}^{2}\left(1-e^{-\pi / \omega T_{0}}\right)}{2 \pi R\left(1+\omega^{2} T_{0}^{2}\right)} P_{0} \\
P_{1, \text { max }}=\frac{2+\omega^{2} T_{0}^{2}\left(1-e^{-\pi / \omega T_{0}}\right)}{\left(1+\omega^{2} T_{0}^{2}\right)\left\{\pi-\omega T_{0}\left(1-e^{-\pi / \omega T_{0}}\right)\right\}} P_{0},
\end{gathered}
$$

where $T_{0}=L / R$.

By calculating using $P_{0}=100 \mathrm{kPa}, f=100 \mathrm{~Hz}, D_{\mathrm{p}}=1 \mathrm{~mm}, \rho=1000 \mathrm{~kg} / \mathrm{m}^{3}, R=$ 2.2 TPa $\cdot \mathrm{s} / \mathrm{m}^{3}$ for the fabricated valve in $\S 3.1$, and $P_{1}=0 \mathrm{kPa}$, no-load flow rates were obtained for the pipe lengths $L_{\mathrm{p}}$, as shown in Fig. 4(b). It was ascertained that the flow rate is decreased only $6 \%$ for $L_{\mathrm{p}}=1 \mathrm{~m}$ because the valve resistance effect is much greater than the inertance effect in this system.

The load characteristics were analyzed for the pipe length $L_{\mathrm{p}}=50 \mathrm{~mm}$. The results are shown in Fig. 4(c). The maximum flow rate $q_{\mathrm{a}, \max }=15 \mathrm{~mm}^{3} / \mathrm{s}$ and the maximum pressure $P_{1, \max }=64 \mathrm{kPa}$ were obtained, which indicates the possibility of the proposed system. 


\section{Fabrication and Experiments of Components}

\subsection{Thin piezoelectric valve}

The proposed thin piezoelectric valve is shown in Fig. 5. The valve consists of a diaphragm with a piezoelectric bimorph disk (piezoelectric diaphragm, hereafter), a valve seat part, and an elastic body, as shown in Fig. 5(a). By applying voltage to the piezoelectric bimorph, the piezoelectric diaphragm deforms upward [Fig. 5(b)] or downward [Fig. 5(c)]. As a result, the valve is opened [Fig. 5(b)] or closed [Fig. 5(c)]. For a large opening, the diameter of the piezoelectric diaphragm cannot be very small, which results in a large pressure-receiving area, and the opening is affected by the pressure. To decrease the pressure-receiving area, in this study, an elastic body is employed, as shown in Fig. 5(a).

The fabricated valve is shown in Fig. 6. The working part is shown in Fig. 6, lower right. The diameter is $7.4 \mathrm{~mm}$ and the thickness is $0.76 \mathrm{~mm}$. The piezoelectric bimorph (T216-A4N-173X, Piezo Systems, Inc.) has a diameter of $6.4 \mathrm{~mm}$, a thickness of 0.41 mm, a maximum displacement of $\pm 4.7 \mu \mathrm{m}$ at $\pm 180 \mathrm{~V}$, a maximum output force of $\pm 2.4 \mathrm{~N}$ at $\pm 180 \mathrm{~V}$, and a resonance frequency of $29 \mathrm{kHz}$. Figure 7 shows the step responses of the piezoelectric bimorph measured in air. On the basis of the approximated curves as the first-order lag responses, the bandwidths of $220 \mathrm{~Hz}$ for step up and $540 \mathrm{~Hz}$ for step down were obtained. The elastic body is a ring-shaped silicone rubber part.

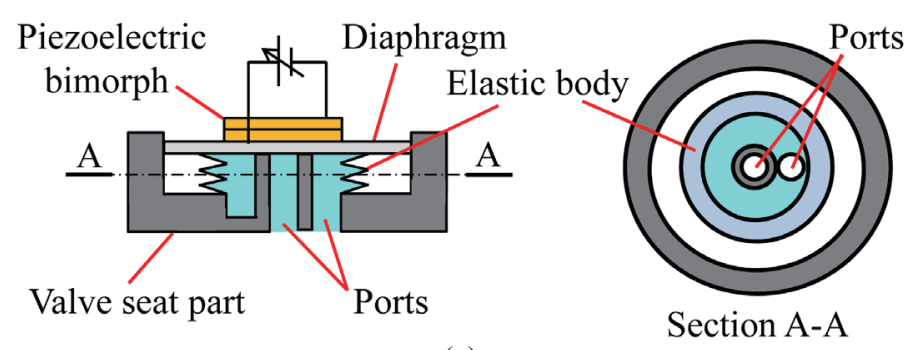

(a)

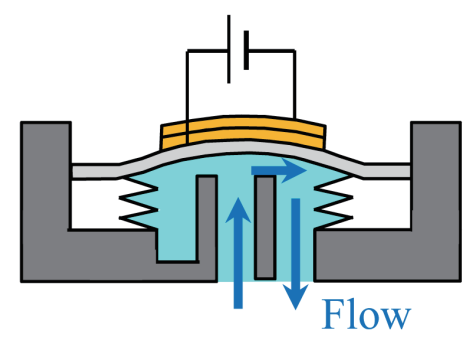

(b)

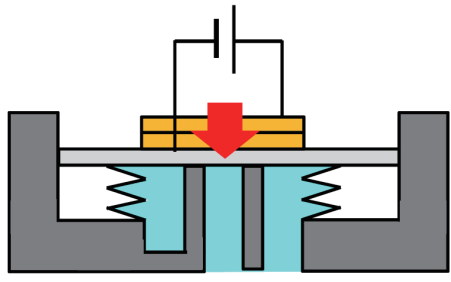

(c)

Fig. 5. (Color online) Proposed thin piezoelectric valve. (a) Structure. (b) Open state. (c) Closed state. 

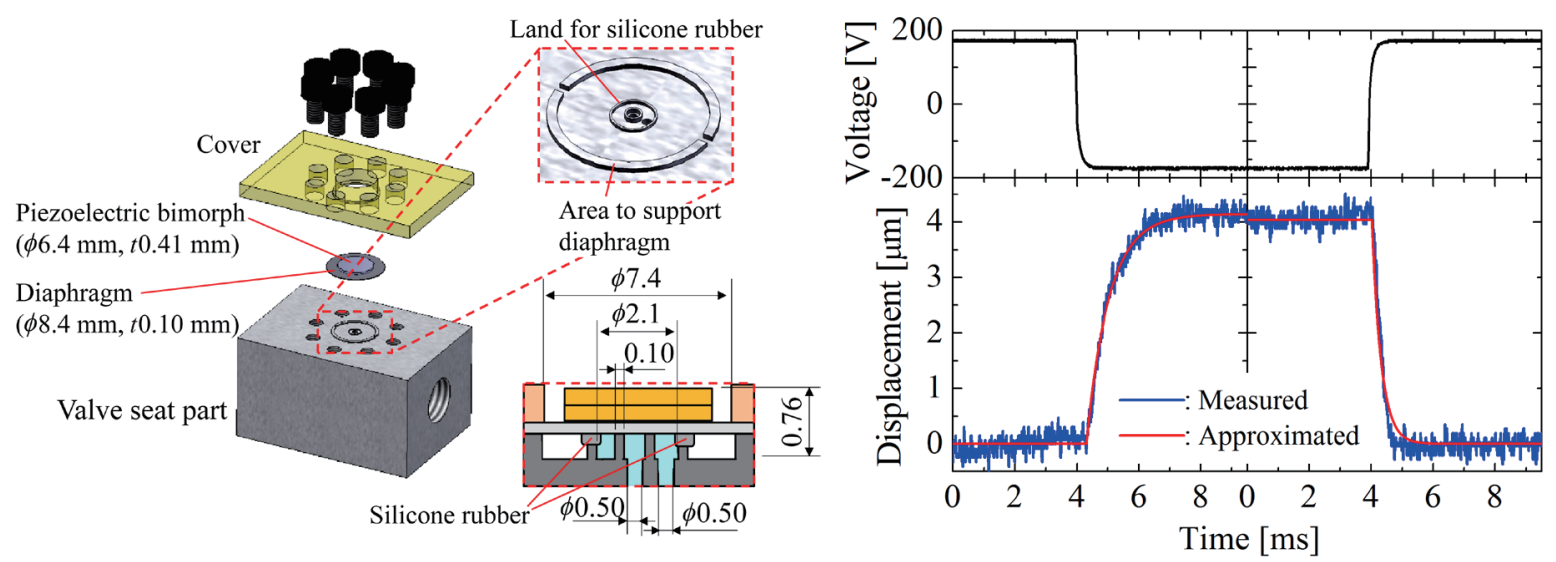

Fig. 6 (left). (Color online) Fabricated thin piezoelectric valve.

Fig. 7 (right). (Color online) Step responses of the piezoelectric diaphragm in air.

The static characteristics of the valve were measured in different flow directions using an experimental setup shown in Fig. 8. Differential pressures were obtained using the measured pressure values $p_{1}$ and $p_{2}$. The flow rates were calculated on the basis of the measured outflow mass in a certain period. The open-state characteristics are shown in Fig. 9. On the basis of the results, the flow resistances were calculated. As a result, it was found that the flow resistance was $2.2 \mathrm{TPa} \cdot \mathrm{s} / \mathrm{m}^{3}$ regardless of the bias pressure (downstream pressure) and flow direction. When the valve is closed, no leakage was observed for $5 \mathrm{~min}$ at an applied pressure of $300 \mathrm{kPa}$.

\subsection{High-frequency piezoelectric pressure source}

A high-frequency piezoelectric pressure source was fabricated, as shown in Fig. 10 , using six piezoelectric diaphragms, which are the same as those used in the thin piezoelectric valves. The overall size is $24 \times 24 \times 25 \mathrm{~mm}^{3}$; however, as the working part is $7.4 \mathrm{~mm}$ in diameter and $6.0 \mathrm{~mm}$ thick, the device can be downsized.

Load characteristics were theoretically analyzed. Assuming that the piezoelectric element can be modeled as a series connection of a displacement source (center displacement $X_{0}$ under no load) and a spring (spring constant $K$ ) and the load is resistive, the following equation is derived:

$$
\left(\frac{P_{0}}{K X_{0} / A}\right)^{2}+\left(\frac{Q_{0}}{N A X_{0} \omega}\right)^{2}=1,
$$

where $A$ and $N$ are the effective sectional area and the number of piezoelectric diaphragms, respectively, $\omega$ is the driving angular frequency, $P_{0}$ is the amplitude of an alternating pressure, and $Q_{0}$ is the amplitude of an alternating flow rate. The derived characteristics are shown in Fig. 11. The maximum amplitude of the alternating pressure 


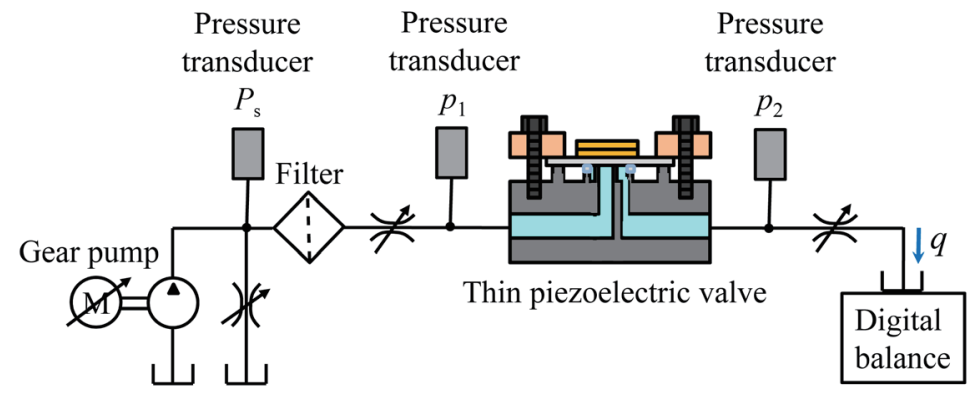

Fig. 8. (Color online) Experimental setup for static characteristics of the thin piezoelectric valve.

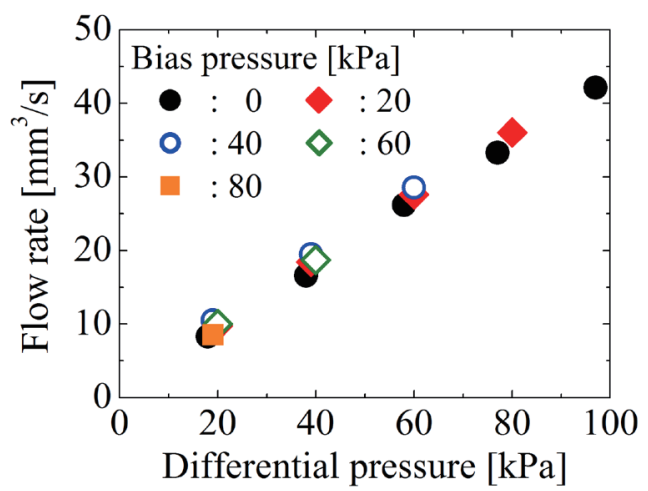

(a)

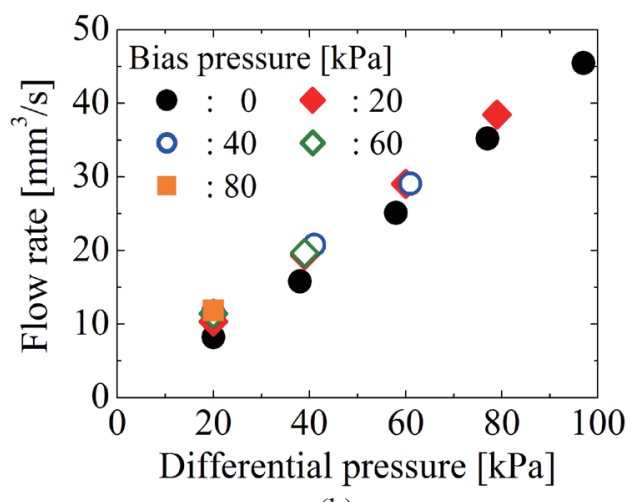

(b)

Fig. 9. (Color online) Measured static characteristics of the thin piezoelectric valve. (a) Inside to outside ports flow. (b) Outside to inside ports flow.
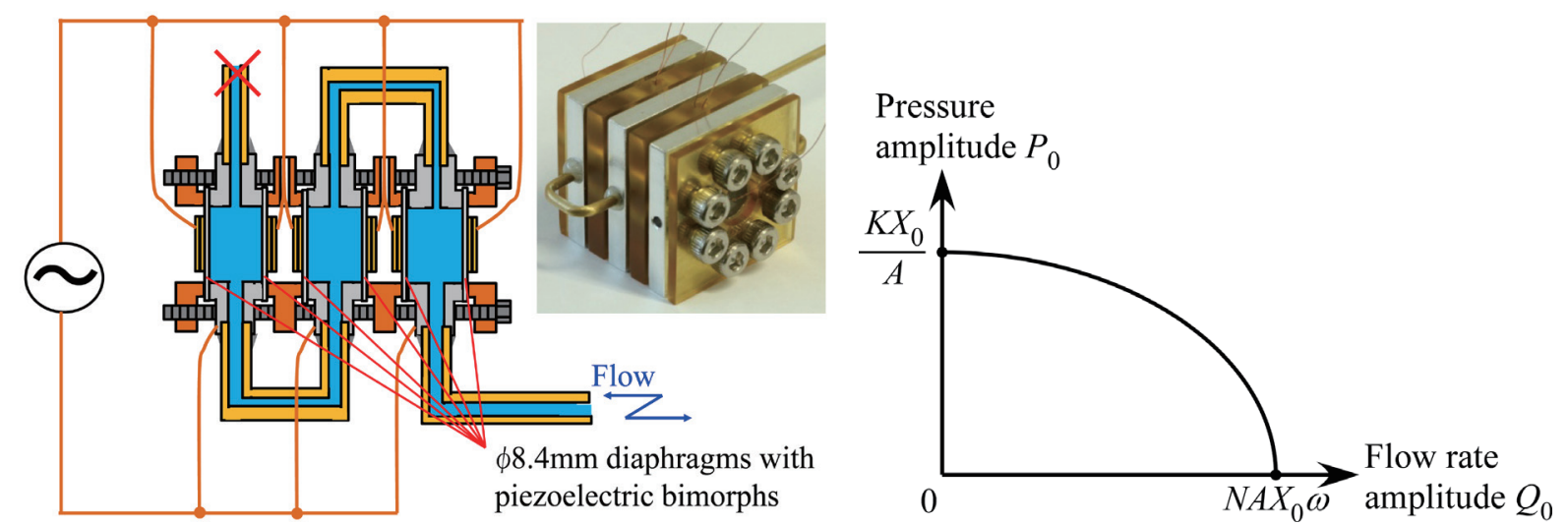

Fig. 10 (left). (Color online) Fabricated piezoelectric high-frequency pressure source.

Fig. 11 (right). Analyzed characteristics of the high-frequency pressure source. 
is $P_{0, \max }=K X_{0} / A$, and the maximum amplitude of the flow rate is $Q_{0, \max }=N A X_{0} \omega$. Owing to an elliptic curve, as a primary approximation, the amplitude of the alternating pressure is independent of the flow rate at low amplitude of the alternating flow rate. In addition, by substituting the characteristic values of the fabricated pressure source into the abovementioned formula, $P_{0, \text { max }}=130 \mathrm{kPa}$ and $Q_{0, \max }=0.26 \mathrm{~cm}^{3} / \mathrm{s}$ were obtained.

\subsection{Bending rubber actuator}

A bending rubber actuator with two chambers was fabricated, as shown in Fig. 12. Each chamber has inner walls to expand axially while constraining the radial expansion when pressurized. ${ }^{(10)}$ The upper and lower parts made of silicone rubber were fabricated by a molding process and they were adhered to the middle silicone rubber sheet. The size of its moving part is $2.0 \times 2.1 \times 10 \mathrm{~mm}^{3}$.

The static characteristics of the bending rubber actuator were measured using air pressure. The relationship between the tip displacement and the differential pressure is shown in Fig. 13. The bias pressure is $50 \mathrm{kPa}$. The maximum tip displacement of 5.0 mm was obtained when 80 and $20 \mathrm{kPa}$ were applied to the chambers.

\section{Experiments using Single-Actuator System}

A single-actuator system using a high-frequency pressure source was fabricated by combining the fabricated components stated in the previous section. The overall view is shown in Fig. 14.

Driving experiments were performed. The high-frequency pressure source was applied to a sinusoidal voltage with an amplitude of $170 \mathrm{~V}$ and a frequency of $100 \mathrm{~Hz}$. The thin piezoelectric valve was applied to a square wave voltage with an amplitude of $170 \mathrm{~V}$, a frequency of $100 \mathrm{~Hz}$, and a phase difference of 0 or $180^{\circ}$ to the high-frequency
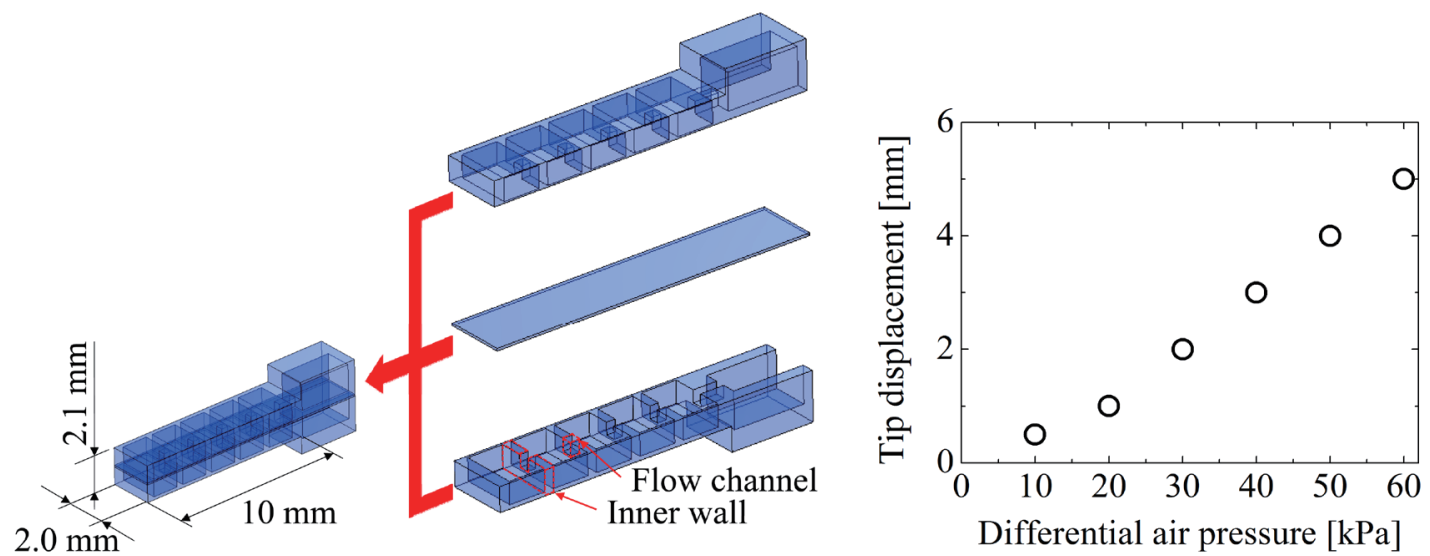

Fig. 12 (left). (Color online) Fabricated bending rubber actuator.

Fig. 13 (right). Measured tip displacement of the bending rubber actuator. 
pressure source driving voltage. The measured motion is shown in Fig. 15. It was found that the bending rubber actuator can bend $4 \mathrm{~mm}$ upward and $3 \mathrm{~mm}$ downward. However, as the openings of the thin piezoelectric valves were not sufficiently large, the maximum tip velocity was $0.1 \mathrm{~mm} / \mathrm{s}$, which should be further improved in the future.

Finally, the maximum output force was measured using a digital balance, as shown in Fig. 16. As a result, $12 \mathrm{mN}$ was obtained at a driving frequency of $100 \mathrm{~Hz}$.

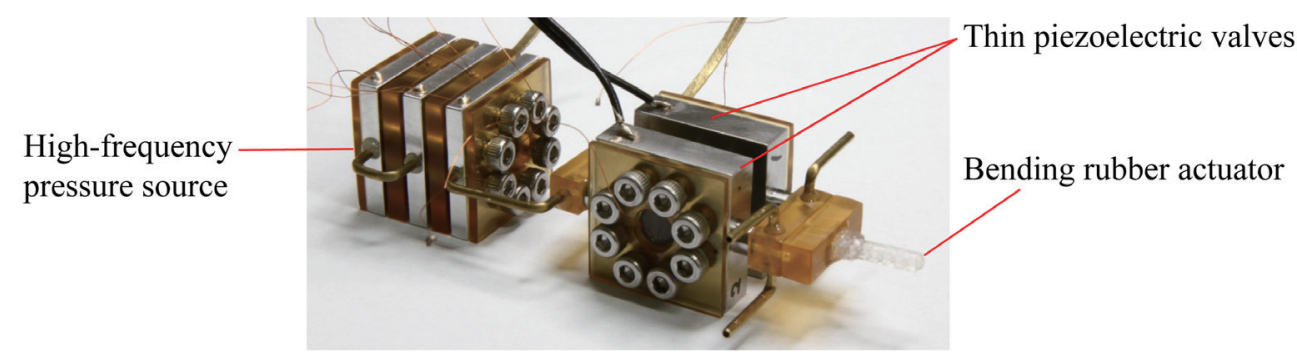

Fig. 14. (Color online) Fabricated single-actuator system.
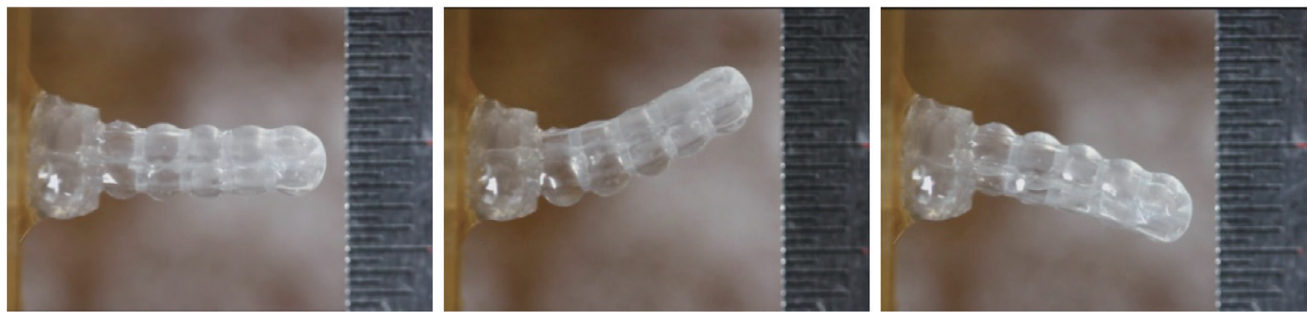

Fig. 15. (Color online) Motion of the bending rubber actuator.

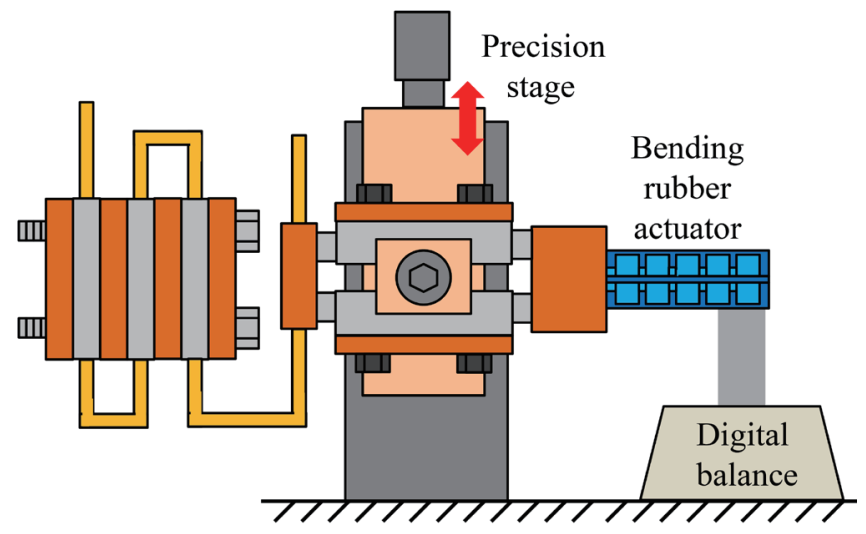

Fig. 16. (Color online) Experimental setup for force measurements. 


\section{Conclusions}

In this paper, a multiple-microactuator system using a high-frequency pressure source was proposed, and a large model with a 10 -mm-long bending rubber actuator was fabricated and experimentally characterized. The main results are summarized as follows:

(1) A multiple-microactuator system using a high-frequency pressure source was proposed and its basic characteristics were clarified through theoretical analyses.

(2) As components of a large model, a thin piezoelectric valve, a high-frequency piezoelectric pressure source, and a 10-mm-long bending rubber actuator were fabricated and their characteristics were experimentally clarified.

(3) A large model system was constructed and its characteristics were experimentally clarified. A maximum tip displacement of $3 \mathrm{~mm}$ and a maximum force of $12 \mathrm{mN}$ were realized.

The characterization of a multiple-actuator system is a future work.

\section{References}

1 M. Takeda: Technical Digest of 14th IEEE Int. Conf. Micro Electro Mechanical Systems 2001 (MEMS 2001) (IEEE, 2001) p. 182.

2 K. Suzumori, T. Miyagawa, M. Kimura and Y. Hasegawa: IEEE/ASME Trans. Mechatron. 4 (1999) 286.

3 Y. Nakazato, Y. Sonobe and S. Toyama: J. Mech. Sci. Technol. I (2010) 51.

4 K. Yoshida, K. Takahashi and S. Yokota: JSME Int. J., B 43 (2000) 29.

5 K. Yoshida and S. Yokota: Preprints 6th Int. Conf. Flow Meas. (FLOMEKO'93) (1993) p. 122.

6 Y. Nishioka, K. Suzumori, T. Kanda and S. Wakimoto: Sens. Actuators, A 164 (2010) 88.

7 A. Kitagawa, S. Jing, C. Liu and H. Tsukagoshi: Proc. 7th JFPS Int. Symp. on Fluid Power, Toyama 2008 (2008) p. 433.

8 T. Miyoshi, K. Yoshida, S. I. Eom and S. Yokota: Sens. Actuators, A 222 (2015) 167.

9 S. Yokota, F. Yajima, K. Takemura and K. Edamura: Adv. Robot. 24 (2010) 1929.

10 K. Yoshida, S. Hara, S. I. Eom and S. Yokota: Proc. 13th Mechatron. Forum Int. Conf. (Mechatronics 2012) (2012) p. 834. 